\title{
Effect of Gold Nanoparticles on Transport Properties of the Protic Ionic Liquid Propylammonium Nitrate
}

\author{
Sandra García-Garabal, Montserrat Domínguez-Pérez, Oscar Cabeza,* Yago Arosa, Luis Miguel Varela, \\ Cristina Fernández-López, Jorge Pérez-Juste, and Isabel Pastoriza-Santos
}

Cite This: J. Chem. Eng. Data 2021, 66, 3028-3037

Read Online

ABSTRACT: Here, we analyze the effect of gold-nanoparticle (AuNPs)-doping of the protic ionic liquid (IL) propyl ammonium nitrate (PAN) on some physical properties, such as density, viscosity, and ionic conductivity, in a broad temperature range. This IL was lightly doped with four different AuNPs (concentration of gold lower than $0.2 \mathrm{mM}$ ): Au nanospheres of about 15, 60 , and $80 \mathrm{~nm}$ diameter and Au nanorods of about $40 \mathrm{~nm}$ length and $12 \mathrm{~nm}$ width. AuNPs were synthesized following a seed growth approach and further functionalized with $\mathrm{O}$-[2-(3-mercapto

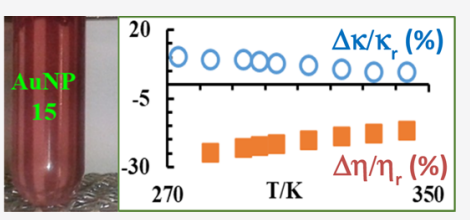
propionylamino)ethyl] $\mathrm{O}^{\prime}$-methyl-poly(ethylene glycol) (m-PEG-SH) in order to provide stability to the IL medium. The stability of the AuNPs in PAN was characterized by UV-vis spectroscopy. The results obtained here indicate that their presence produces a strong influence on the measured transport properties of PAN, decreasing viscosity up to $25 \%$ and increasing ionic conductivity up to $10 \%$ for the smallest AuNPs. Moreover, as temperature increases, the influence of AuNPs produces a decrease in the percentage deviations for viscosity and ionic conductivity. In the case of density, the AuNP-doping produces a mainly unexpected increase only for the sample with the smallest AuNPs, that is practically constant with temperature. When we equalize the AuNP concentration for all sizes and shapes, it is observed that the smallest AuNPs give higher variations in the studied properties than the bigger ones; also, the shape of the rod has an influence on the measured results. In addition, we have applied Walden's rule to relate the three measured magnitudes, observing that AuNP addition decreases ionicity with respect to the reference sample, which is even more when we added smaller AuNPs. Finally, we explain all data assuming cation solvation of the AuNPs, which creates a local breaking of the pseudolattice structure of the PAN. Comparison with other published data is difficult because the behavior of a nanoparticle solution strongly depends on its particular composition and no data are available, in our knowledge, of any similar mixture to those studied here.

\section{INTRODUCTION}

Nowadays, we can find in the literature several references about the potential uses of nanofluids (NFs) in diverse fields such as heat transfer and cooling technologies, ${ }^{1-3}$ solar thermal engineering systems, ${ }^{4-6}$ biomedicine, ${ }^{7-10}$ or optical applications. ${ }^{11,12} \mathrm{~A} \mathrm{NF}^{13}$ is a fluid that consists of a base fluid (a common molecular solvent such as water, glycols, etc.) with a suspension of solid nanometer (NP)-size particles (generally carbon, a metal, or a metal oxide). The addition of small amounts of these nanoparticles produces changes in the physical properties of the base fluid thus tailoring it for their future applications.

One of the most studied properties of NFs is thermal conductivity. ${ }^{14-17}$ This is because these fluids have shown a higher value compared to the base fluid, making them appropriate for substituting traditional coolants. This enhancement is affected by the NPs' nature (metallic or non-metallic) as well as their size and shape. ${ }^{14-17}$

However, apart from thermal conductivity, there are other properties that play an important role in the features of any NF such as viscosity, ${ }^{16,18-21}$ density, ${ }^{16,18,19}$ or ionic conductivity $^{18,22}$ that will also affect its behavior. Thus, viscosity is important because not only does it have an influence on the fluid flow but also on the heat-transport properties and consequently the thermal conductivity. In the literature, the majority of articles are focused on the development of theoretical models to predict viscosity in NFs or to analyze the effect of several factors such as shape/size of particle, temperature, $\mathrm{pH}$, and so forth on the viscosity. ${ }^{18-21}$ However, neither has developed an appropriate theoretical model that can explain the behavior of the NF nor has found a suitable explanation about the effect of the factors mentioned above on the viscosity. ${ }^{20,21}$ This is because classical models have been used to describe the behavior of NFs when it is well known that these systems do not follow that behavior, and so, many of the mentioned studies are not consistent because of contradictory results. Due to these discrepancies, the gathering of experimental results is necessary in order to contribute to the development of new theoretical models as well as to

Received: February 22, 2021

Accepted: July 7, 2021

Published: July 21, 2021 
Table 1. Name, Provenance, CAS Number, Molar Mass, and Mass Fraction Purity of the Chemicals

\begin{tabular}{|c|c|c|c|c|c|}
\hline chemical name & provenance & CAS number & $\begin{array}{c}\text { molar } \\
\text { mass/g mol-1 }\end{array}$ & $\begin{array}{l}\text { mass fraction } \\
\text { purity }\end{array}$ & analysis method \\
\hline PAN & Io-li-tec & $22113-88-8$ & 122.12 & $>0.97$ & NMR \\
\hline tetrachloroauric acid $\left(\mathrm{HAuCl}_{4} \times 3 \mathrm{H}_{2} \mathrm{O}\right)$ & Aldrich & $16961-25-4$ & 393.83 & 0.99 & $\begin{array}{l}\text { trace metal } \\
\text { analysis }\end{array}$ \\
\hline $\begin{array}{l}\mathrm{O} \text {-[2-(3-mercaptopropionylamino)ethyl] } \\
\mathrm{O}^{\prime} \text {-methyl-poly(ethylene glycol) (m-PEG-SH) }\end{array}$ & Fluka & $401916-61-8$ & 5000 & 1 & NMR \\
\hline CTAB $\mathrm{CH}_{3}\left(\mathrm{CH}_{2}\right)_{15} \mathrm{~N}(\mathrm{Br})\left(\mathrm{CH}_{3}\right)_{3}$ & Fluka & $57-09-0$ & 364.45 & $\geq 0.98$ & TLC \\
\hline silver nitrate $\left(\mathrm{AgNO}_{3}\right)$ & Sigma & $7761-88-8$ & 169.81 & $\geq 0.99$ & $\begin{array}{l}\text { titration by } \\
\text { KSCN }\end{array}$ \\
\hline sodium borohydride $\left(\mathrm{NaBH}_{4}\right)$ & Sigma & $16940-66-2$ & 37.83 & 0.99 & $\begin{array}{l}\text { hydrogen } \\
\text { evolution }\end{array}$ \\
\hline sodium citrate $\left(\mathrm{C}_{6} \mathrm{H}_{5} \mathrm{O}_{7} \mathrm{Na}_{3} \times 2 \mathrm{H}_{2} \mathrm{O}\right)$ & Sigma & $6132-04-3$ & 294.10 & $\geq 0.99$ & $\begin{array}{l}\text { titration by } \\
\mathrm{HClO}_{4}\end{array}$ \\
\hline ethanol & Sigma & $64-17-5$ & 46.07 & $\geq 0.998$ & GC \\
\hline
\end{tabular}

understand the effect that the addition of nanoparticles has on the physical properties of a certain fluid.

A particular case of NFs is the ioNanofluids (INFs), ${ }^{23}$ whose base component is an ionic liquid (IL), making them more complex than those that use a common molecular solvent due to specific interactions between the ions and the nanoparticles. It makes understanding of their physical properties even more difficult. In spite of this, scientists should make special efforts in the study and comprehension of these systems due to their promising possibilities.

Focused on INFs, in the literature, some data exists for thermal conductivity and viscosity but data for density and ionic conductivity hardly exists. However, the study of the ionic conductivity in this kind of compounds is necessary from the theoretical and practical points of view. For example, in the case of batteries, the role and importance of the electrolytes used in this kind of devices are well known. The common electrolytes employed in batteries include, among other advantages, high energy density, low self-discharge, and longer lifespan; however, their safety problems (that can result in short circuits and even fire), as well as their cost and environmental impact make necessary the search for new ones. Among the candidates being considered as alternatives to typical electrolytes are polymer electrolytes, ${ }^{24} \mathrm{ILs}$, and INFs, some of them with nanoparticle hybrids. ${ }^{25-29}$

Herein, we prepare stable INFs based on the combination of synthesized gold nanoparticles (AuNPs) dispersed in ethanol and propyl ammonium nitrate (PAN). The final INF contains the AuNPs dispersed in PAN with $2 \%$ in volume of ethanol (which represents a mole fraction of ethanol of 0.0365 ). This last binary mixture will be considered as the reference sample or base fluid. The choice of AuNPs to prepare an INF based on the protic IL PAN was due to several reasons: (1) nanoparticles of control size and shape can be obtained following wet chemical methods, (2) the surface chemistry of the AuNPs can be tailored through thiol chemistry, and (3) the stability of the resulting INF can be monitored through the optical properties of the AuNPs. ${ }^{15}$ Moreover, we study the effect of AuNPs in the density, viscosity, and ionic conductivity of the reference sample in a broad temperature range from 278 to $343 \mathrm{~K}$. Additionally, we will analyze the effect of AuNP size and shape on the physical properties of the studied INFs after calculating the different magnitudes for common gold content.

\section{MATERIALS AND METHODS}

2.1. Chemical Products and Nanoparticles. 2.1.1. Chemicals. Tetrachloroauric acid $\left(\mathrm{HAuCl}_{4} \times 3 \mathrm{H}_{2} \mathrm{O}\right)$, perchlorate was supplied by Aldrich. O-[2-(3Mercaptopropionylamino)ethyl] $O^{\prime}$-methyl-poly(ethylene glycol) (m-PEG-SH, $M_{\mathrm{w}}$ 5000) and cetyltrimethylammonium bromide (CTAB) were purchased from Fluka. Silver nitrate $\left(\mathrm{AgNO}_{3}\right)$, sodium borohydride $\left(\mathrm{NaBH}_{4}\right)$, citric acid $\left(\mathrm{C}_{6} \mathrm{H}_{5} \mathrm{O}_{7} \mathrm{Na}_{3} \times 2 \mathrm{H}_{2} \mathrm{O}\right)$, and ascorbic acid were procured from Sigma. $\mathrm{HCl}(37 \%)$ was supplied by Panreac. PAN with mass fraction purity $>0.97$ was provided by IoLiTec. In addition, we used pure grade ethanol and Milli-Q grade water in all preparations. The chemicals used in this study with the name, provenance, CAS number, molar mass, and purity are presented in Table 1.

2.1.2. AuNP Synthesis. 2.1.2.1. AuNP15. Citrate-stabilized $\mathrm{Au}$ spheres of around $14 \mathrm{~nm}$ diameter were synthesized as previously stated. ${ }^{30,31}$ Briefly, $5 \mathrm{~mL}$ of trisodium citrate $(0.5 \%)$ was added to $95 \mathrm{~mL}$ of a boiling aqueous $\mathrm{HAuCl}_{4}$ solution $(0.5$ $\mathrm{mM}$ ). The gold nanospheres obtained were around $13.9 \pm 2.0$ $\mathrm{nm}$ in diameter as we can observe in Figure 1A, where we show transmission electron microscopy (TEM) pictures of AuNP15, and in Figure $1 \mathrm{~B}$, the histogram giving the size distribution.

2.1.2.2. AuNP60 and AuNP80. Average-sized, 60 and $80 \mathrm{~nm}$ in diameter CTAB-stabilized gold nanospheres were prepared following the seeded growth method mentioned previously. $^{30,31}$ In short, to $100 \mathrm{~mL}$ of an aqueous solution consisting of $\mathrm{HAuCl}_{4}(0.5 \mathrm{mM})$ and $\mathrm{CTAB}(0.015 \mathrm{M})$ at 308 $\mathrm{K}$, was added $1 \mathrm{~mL}$ of ascorbic acid $(0.1 \mathrm{M})$, followed by the addition of citrate-stabilized $\mathrm{Au}$ seeds of $15 \mathrm{~nm}$ in a final concentration of $[\mathrm{Au}]=3.97 \times 10^{-6} \mathrm{M}$ in terms of $\mathrm{Au}$ atoms. It was allowed to react for $3 \mathrm{~h}$. The gold nanospheres obtained were around $67.0 \pm 8.3 \mathrm{~nm}$ in diameter as we can observe in Figure 1C, where we show TEM pictures of AuNP60, and in Figure $1 \mathrm{D}$, the histogram giving the size distribution. To obtain $80 \mathrm{~nm}$ particles, the $60 \mathrm{~nm}$ particles were overgrown by means of the same procedure described for the growth of $15 \mathrm{~nm}$ AuNPs. The gold nanospheres obtained were around $81.2 \pm$ $7.8 \mathrm{~nm}$ in diameter as we can observe in Figure 1E, where we show TEM pictures of AuNP80, and in Figure 1F, the histogram giving the size distribution. All resulting AuNPs were centrifuged at $6000 \mathrm{rpm}$ for $45 \mathrm{~min}(15 \mathrm{~nm}), 20 \mathrm{~min}(60$ $\mathrm{nm})$, and $10 \mathrm{~min}(80 \mathrm{~nm})$ and then redispersed in the same volume of water.

2.1.2.3. AuNProd. For the synthesis of the gold nanorods, a previously stated strategy was followed. ${ }^{31,32}$ First, $2-3 \mathrm{~nm}$ AuNPs were synthesized by adding $0.05 \mathrm{~mL}$ of freshly prepared $25 \mathrm{mM}$ borohydride to $5 \mathrm{~mL}$ of $0.25 \mathrm{mM} \mathrm{HAuCl}_{4}$ in an aqueous $0.1 \mathrm{M} \mathrm{CTAB}$ solution under vigorous stirring. After $2 \mathrm{~h}, 24 \mu \mathrm{L}$ of Au seeds was added to a growth solution 

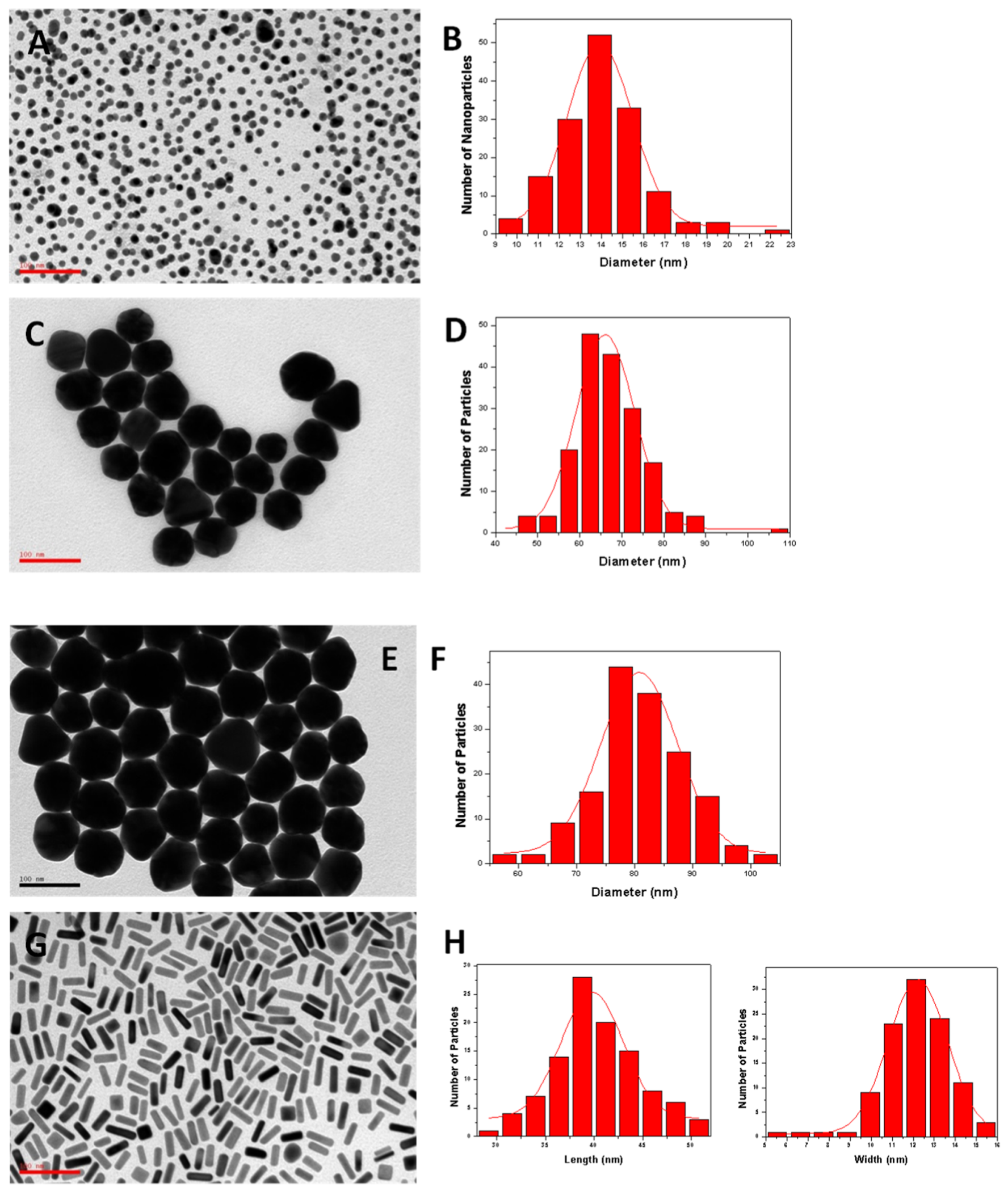

Figure 1. (A-G) TEM images of Au nanospheres and corresponding histograms of $13.9 \pm 2.0(\mathrm{~A}, \mathrm{~B}), 67.0 \pm 8.3(\mathrm{C}, \mathrm{D})$, and $81.2 \pm 7.8 \mathrm{~nm}(\mathrm{E}, \mathrm{F})$ in diameter and $\mathrm{Au}$ nanorods of $40.4 \pm 4.4$ in length and $12.0 \pm 1.5$ in width $(\mathrm{G}-\mathrm{H})$.

containing $0.1 \mathrm{M} \mathrm{CTAB}, 0.5 \mathrm{mM} \mathrm{HAuCl}, 0.12 \mathrm{mM} \mathrm{AgNO}_{3}$, $0.8 \mathrm{mM}$ ascorbic acid, and $19 \mathrm{mM}$ HCL. It was allowed to react for $12 \mathrm{~h}$ and then the colloids were centrifugated at 6000 $\mathrm{rpm}$ for $30 \mathrm{~min}$ and redispersed in the same volume of water. The gold nanorods obtained were around $40.4 \pm 4.4 \mathrm{~nm}$ in length $(l)$ and $12.0 \pm 1.5 \mathrm{~nm}$ in width $(w)$ as we can observe in Figure 1G, where we show TEM pictures of AuNProd, and in Figure $1 \mathrm{H}$, the histograms giving both size distributions. The AuNP characterization is improved in Section 3.1. 
2.1.3. $m P E G-S H$ Functionalization and PAN Transfer. First, an aqueous solution containing mPEG-SH (amount to provide 4 molecules $/ \mathrm{nm}^{2}$ ), previously sonicated for $15 \mathrm{~min}$, was added dropwise under vigorous stirring to $10 \mathrm{~mL}$ of the obtained Au nanoparticles. $\left([\mathrm{Au}]=5 \times 10^{-4} \mathrm{M}\right)$. After $1 \mathrm{~h}, \mathrm{Au}$ nanoparticles were centrifuged twice (at $6000 \mathrm{rpm}$ for $30 \mathrm{~min}$ ) to remove the excess PEG, and they were redispersed in a small volume of ethanol. The final concentration of the different Au colloids in ethanol was 6.5, 9.0, 10.5, and 7.5 mM in terms of $\mathrm{Au}$ atoms for 15, 60, and $80 \mathrm{~nm} \mathrm{Au}$ nanospheres and $\mathrm{Au}$ nanorods, respectively. The PAN transfer was carried out by adding with vigorous stirring, $200 \mu \mathrm{L}$ of Au colloids in ethanol to $9.8 \mathrm{~mL}$ of PAN. The uncertainty for the concentration of nanoparticles is $0.01 \mathrm{Mm}$.

2.2. Experimental Techniques. 2.2.1. Nanoparticle Characterization. UV-vis-NIR spectra were measured with an Agilent 8453 UV-vis spectrophotometer using a cell with a $1 \mathrm{~cm}$ path length. The wavelength accuracy of the spectrophotometer is $< \pm 0.5 \mathrm{~nm}$. TEM was carried out with a JEOL JEM 1010 transmission electron microscope operating at an acceleration voltage of $100 \mathrm{kV}$. The TEM has a resolution of $0.4 \mathrm{~nm}$. We measured the refractive index at the sodium D line with a commercial Abbe refractometer, Atago DR-M2, with a temperature controller (resolution $0.1 \mathrm{~K}$ at $298 \mathrm{~K}$ ). The refractometer was calibrated with deionized water, and its expanded uncertainty ( 0.95 level of confidence) at the sodium line was $4.4 \times 10^{-4}$.

2.2.2. Density and Viscosity. Density and dynamic viscosity measurements were carried out in an Anton Paar SVM 3000 Stabinger viscodensimeter, which was thermostated with an internal Peltier cell with a standard uncertainty of $0.02 \mathrm{~K}$. The expanded uncertainty in the measurement of density is about $0.5 \mathrm{~kg} \mathrm{~m}^{-3}$, while for viscosity, the relative standard uncertainty is 0.004 of the measured value. This viscodensimeter automatically corrects the density value depending on the viscosity of the sample. Also, the viscosity value range goes from 0.2 up to $20,000 \mathrm{mPa}$ s and is checked with reference chemicals recommended by the supplier (M114 and C117 SH Calibration Service GmbH).

Remember that the viscosity of INFs varies exponentially with temperature changing its value more than 10 times in the temperature range studied, from 278 to $343 \mathrm{~K}$. Note that above $363 \mathrm{~K}$, AuNPs precipitate.

2.2.3. Ionic Conductivity. Ionic conductivities were measured using a Crison GLP31 conductimeter, which has a relative standard uncertainty of less than 0.005 . This conductimeter uses a fixed $500 \mathrm{~Hz}$ ac current of $4.5 \mathrm{~V}$ peak to peak. The samples were thermostated in an external bath with an uncertainty of $0.1 \mathrm{~K}$. Details of the ionic conductivity measurement and calibrated procedure were published previously. ${ }^{33}$ The reference fluids used to calibrate the measurement instrument were those called as $\mathrm{P} / \mathrm{N}$ L7W9710.99 and P/N L7W9700.99. Measurements were carried out at atmospheric pressure in a broad temperature range, from 273 to $353 \mathrm{~K}$.

\section{RESULTS AND DISCUSSION}

3.1. AuNP Characterization. Au nanospheres of $13.9 \pm$ 2.0 (AuNP15), $67.0 \pm 8.3$ (AuNP60), and $81.2 \pm 7.8 \mathrm{~nm}$ (AuNP80) diameter and Au nanorods of $40.4 \pm 4.4$ length (l) and $12.0 \pm 1.5 \mathrm{~nm}$ width $(w)$ (AuNProd) were synthesized in water, following the wet chemical methods (see Experimental part for details). As presented above, Figure 1 shows the TEM images of the different AuNPs and the corresponding histograms giving the size distribution.

The particles, initially in water, were transferred to ethanol, but it a ligand exchange with mPEG-SH was required. This molecule contains a thiol group with a strong affinity for gold that is able to displace the capping agent (citrate or CTAB) and to bind covalently to the metal surface. Once the AuNPs were in ethanol, they could be easily redispersed in PAN by simple addition. The final colloids in PAN contained $(2 \pm$ 0.05 )\% of ethanol in volume (equivalent to a mole fraction of ethanol $\left.x_{\text {et }}=0.0365 \pm 0.0005\right)$. As shown in Figure $S 1$ in the Supporting Information for $60 \mathrm{~nm}$ AuNPs, their transfer to PAN just produced a slight red-shift of the localized surface plasmon resonance band due to the higher refractive index of PAN $\left(n_{\mathrm{D}}=1.4551\right)$ with respect to that of ethanol $\left(n_{\mathrm{D}}=\right.$ $1.3612)$, purity $99.8 \%$ measured at the same temperature 298 $\mathrm{K}$. A similar behavior was observed for the rest of the samples (data not shown). As observed in Figure 2, the resulting INFs

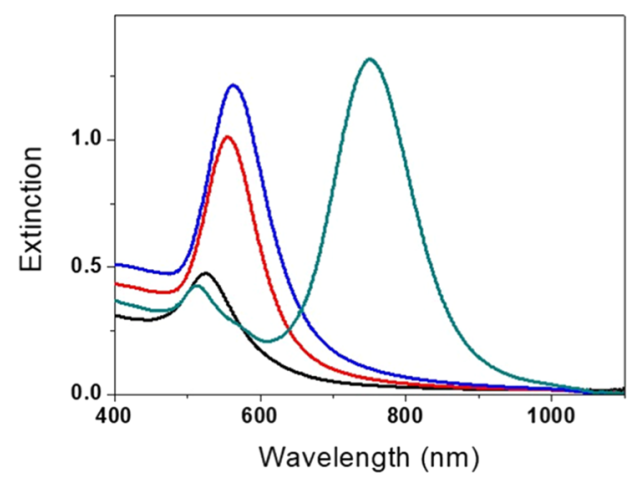

Figure 2. Extinction spectra of different INFs based on PANdispersed AuNPs: $15 \mathrm{~nm} \mathrm{Au} \mathrm{nanospheres} \mathrm{(black} \mathrm{line),} 60 \mathrm{~nm} \mathrm{Au}$ nanospheres (red line), $80 \mathrm{~nm} \mathrm{Au}$ nanospheres (blue line), and $40 \pm$ $12 \mathrm{~nm} \mathrm{Au}$ nanorods (green line).

exhibit the characteristic optical features in the extinction spectra of their dispersed AuNPs, shown pink (wavelength 550 $\mathrm{nm}$ ) for the spheres (as seen in TOC) and maroon (wavelength $800 \mathrm{~nm}$ ) for the one with rods. Therefore, the fact that the optical properties of INFs are just slightly changed with respect to those of AuNPs in ethanol demonstrates that mPEG-SH provides good colloidal stability to the AuNPs in $\mathrm{PAN}$. The concentrations of the different $\mathrm{Au}$ dispersions in terms of $\mathrm{Au}$ metal were $0.13 \mathrm{mM}$ (AuNP15), $0.18 \mathrm{mM}$ (AuNP60), $0.21 \mathrm{mM}$ (AuNP80), and $0.15 \mathrm{mM}$ (AuNProd). The refractive index of all samples was the same within the experimental uncertainties, $n_{\mathrm{D}}=1.4516$, on average, with no influence of the AuNPs up to the fourth decimal. The discrepancy between the refractive index of pure PAN and of those which contain nanoparticles $\left(\Delta n_{\mathrm{D}} \approx 2 \times 10^{-3}\right)$ is caused by the ethanol concentration inherent to the process. In order to verify it, we prepared solutions of PAN + ethanol obtaining the same refractive index as their counterparts that contain ethanol. A summary of the four INFs prepared is given in Table 2, where we include the particles' characteristic lengths, the gold concentration, the estimated number of AuNPs, and the volume and external surface of all AuNPs. For the last three calculations, we assume that the density of the AuNPs is the same as that of gold. Obviously, the values calculated have very high uncertainty, and they are intended simply to illustrate a qualitative analysis of the obtained results. 
Table 2. Mean Characteristics of the INF Samples Solved in PAN + 2 vol \% Ethanol (i.e., $\left.x_{\text {et }}=0.0365\right)$, including, from Left to Right, Size of AuNPs; Gold Concentration in Mole per Liter of Solution (C), Mole per Kilogram of Solvent $\left(C^{\prime}\right)$, Mole Fraction of Gold; AuNPs Number per Liter of Solution ( $n)$; AuNPs Surface per Liter of Solution $(S)$, and AuNP Volume per Liter of Solution $(V)^{a}$

\begin{tabular}{llccccccc}
\multicolumn{1}{c}{ sample } & \multicolumn{1}{c}{ AuNP size $/ \mathrm{nm}$} & $\mathrm{C} / \mathrm{mM}$ & $\mathrm{C}^{\prime} / \mathrm{m} \times 10^{-3}$ & $x_{\mathrm{Au}} \times 10^{-4}$ & $w \%$ gold & $n_{\text {AuNP }} / \mathrm{L} \times 10^{12}$ & $S / \mathrm{m}^{2}$ & $V / \mathrm{m}^{3} \times 10^{-9}$ \\
AuNP15 (spherical) & $13.9 \pm 2.0$ & 0.13 & 0.91 & 1.11 & 0.0179 & 943 & 0.57 & 1.33 \\
AuNP60 (spherical) & $67.0 \pm 8.3$ & 0.18 & 1.26 & 1.53 & 0.0247 & 11.7 & 0.16 & 1.84 \\
AuNP80 (spherical) & $81.2 \pm 7.8$ & 0.21 & 1.46 & 1.79 & 0.0288 & 7.64 & 0.16 & 2.14 \\
AuNProds (rods) & $40.4 \pm 4.4(l) 12.0 \pm 1.5(w)$ & 0.15 & 1.05 & 1.28 & 0.0206 & 335 & 0.59 & 1.53
\end{tabular}

${ }^{a}$ Standard uncertainty: $u(C)=0.01 \mathrm{mM}, u\left(C^{\prime}\right)=7 \times 10^{-5} \mathrm{~m}, u\left(x_{\mathrm{Au}}\right)=9 \times 10^{-6}, u(w \%)=1.4 \times 10^{-3}$, and $u\left(x_{\mathrm{et}}\right)=0.0005$. Relative standard uncertainty: $u_{\mathrm{r}}(n)=0.5, u_{\mathrm{r}}(S)=0.5$ and $u_{\mathrm{r}}(V)=0.5$ (given for qualitative analysis).

3.2. Density. Density data measured for the four different INFs studied in the function of temperature are plotted in Figure 3 and the corresponding numerical data is presented in

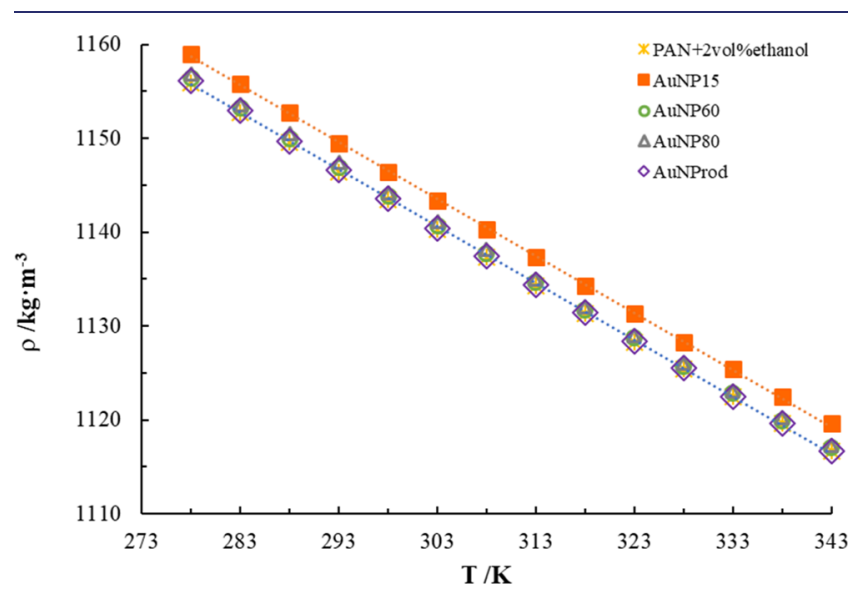

Figure 3. Measured density of reference sample, PAN +2 vol \% ethanol (star) and different INFs based on PAN-dispersed AuNPs: AuNP15 (square), AuNP60 (circle), AuNP80 (triangle), and AuNProd (rhombus). Lines are the best fit of the AuNP15 and AuNProd samples to a straight line.

Table S1, including the reference sample (PAN with 2 vol \% in ethanol, i.e., a mole fraction of ethanol equal to $x_{\mathrm{et}}=0.0365$ ). As can be observed, density presents a linear behavior with temperature in the range studied, and its value decreases as temperature increases with a very similar slope for the four samples, about $-0.7 \mathrm{mg} \mathrm{cm}^{-3} \mathrm{~K}^{-1}$. However, if we analyze the values obtained at the same temperature, the sample AuNP15 presents a much higher density than the base fluid, while the other INFs have a similar density as that of the reference sample within the experimental uncertainties. To better observe density deviations, we calculate the increase in the percentage of the density of the four INFs with respect to the reference sample at all temperatures. Results are plotted in Figure 4, where we observe how the density value increases $0.25 \%$ in the AuNP15 sample and very subtly (less than $0.1 \%$ ) in the rest of the samples which is within the experimental uncertainties. This effect slightly decreases with temperature.

The small increase in density of the INF with AuNP15 is very high compared with that expected in the mixture of the reference liquid and the AuNPs. Thus, if we perform the corresponding calculations, based on the mass and volumes of the AuNPs added to the sample, the density variation would be below the experimental uncertainties of our equipment as it happens for the three other INFs. Take into account that gold represents only tenths of ppm in weight of the sample and

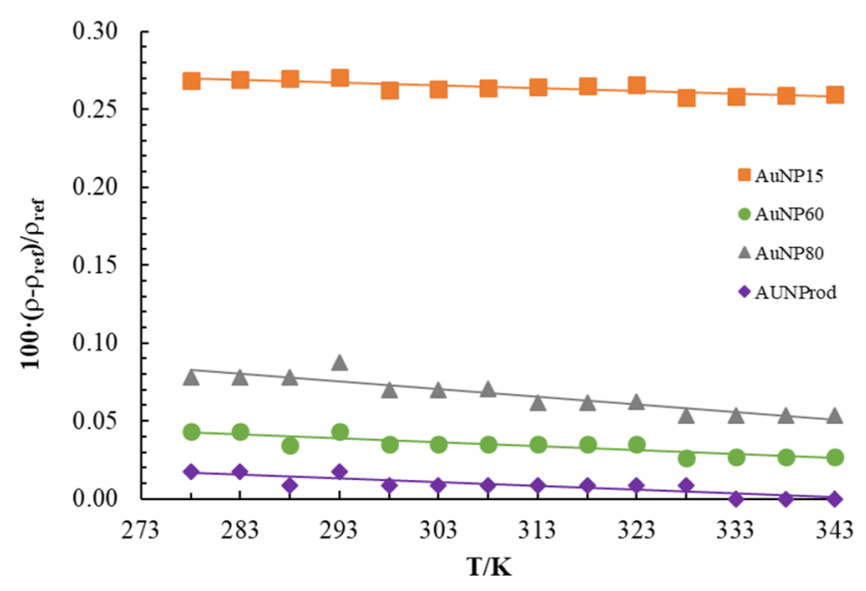

Figure 4. Percentage deviation of density vs temperature for the different INFs based on PAN-dispersed AuNPs with regard to the reference sample: AuNP15 (square), AuNP60 (circle), AuNP80 (triangle), and AuNProd (rhombus). Lines are the best fit of the data to a straight line.

units of ppm of volume fraction. One explanation for the observed increase in density is that AuNP15 are solvated by the propyl ammonium cation, thus packing tighter in the solvation layer than they were in the bulk. Although some authors in the literature report that AuNPs can be solvated by anions ${ }^{25}$ or cations, ${ }^{34,35}$ in our case, we assume that the AuNPs were solvated by the cations due to their surfactant nature. This model is validated by the fact that only the smallest spherical AuNPs present a density increase because they have a greater surface to be solvated, while the external surface in the other two spherical AuNPs is more than 3 times smaller, as observed in Table 1. The AuNProd is a special case because it presents a similar external surface to that of the INF with AuNP15, while its density is not increased at all. Thus, it seems that the spherical shape allows a better cation packing than the rod one.

3.3. Viscosity. The experimental dynamic viscosity data for the compounds studied versus temperature are presented in Table S1 and illustrated in Figure 5. As it was expected, ${ }^{14,36}$ viscosity values decrease markedly with temperature increases, following a Vogel-Tamman-Fulcher (VFT) trend. ${ }^{37-39}$ The VFT equation can be written as

$$
\eta=\eta_{\infty} \exp \left\{\frac{B_{\eta}}{T-T_{\eta}}\right\}
$$

where $\eta_{\infty}, B_{\eta}$, and $T_{\eta}$ are treated as fitting parameters. The first one represents the limiting viscosity value at infinite temperature, the second one is related to a pseudo activation energy, 


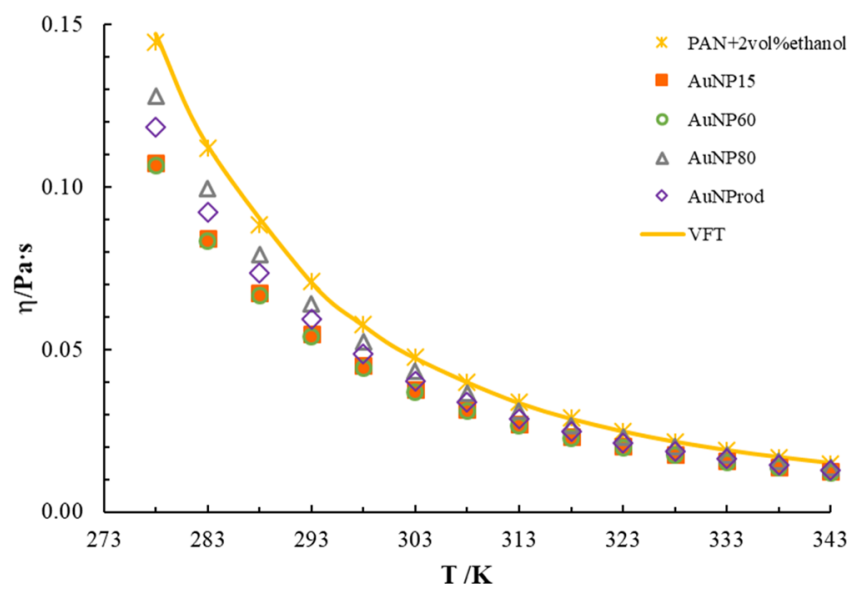

Figure 5. Measured viscosity vs temperature of reference sample, PAN +2 vol \% ethanol (star symbols), and different INFs: AuNP15 (square), AuNP60 (circle), AuNP80 (triangle), and AuNProd (rhombus). The line is a VFT-type equation fitted to the reference sample data.

while $T_{\eta}$ is usually near but above the glass transition temperature.

Viscosity clearly decreases for the INFs studied with respect to that of the reference sample, the values obtained being $25 \%$ (AuNP15 and AuNP60), 10\% (AuNP80), and 15\% (AuNProds) lower with respect to the reference sample.

To compare the viscosity measured in the different INFs among them, we must normalize the data to a common concentration, accepting a linear dependence of viscosity decrease with respect to concentration, that is, $\Delta \eta \alpha C$, in the range of concentrations studied, as was suggested by other authors. $^{20,21,40}$ Thus, in Figure 6, we present the percentage

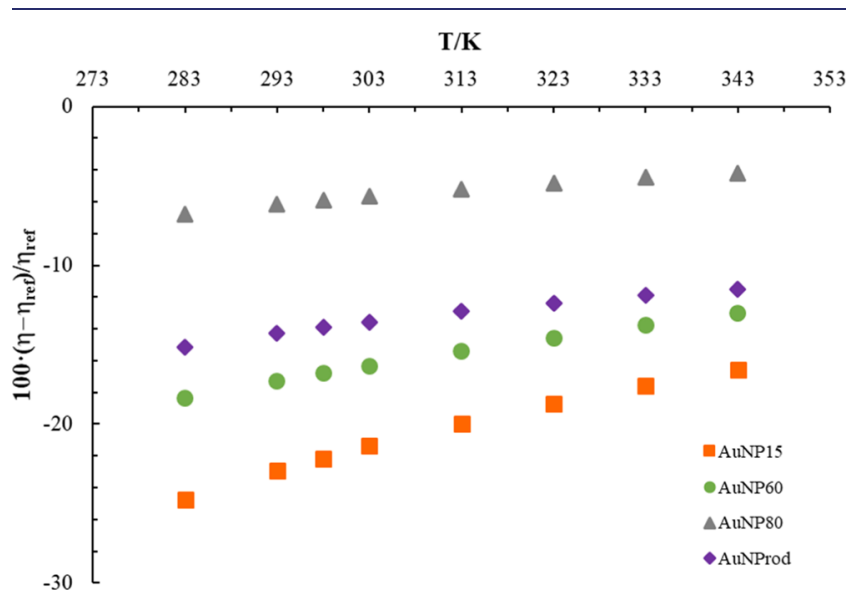

Figure 6. Percentage deviation of viscosity against temperature for the different INFs studied normalized to the same concentration $(0.13$ $\mathrm{mM})$ corresponding to the AuNP15 samples: AuNP15 (square), AuNP60 (circle), AuNP80 (triangle), and AuNProd (rhombus).

deviation of viscosity for the four INFs studied with respect to that of the reference fluid versus temperature, after being normalized to the same concentration $(0.13 \mathrm{mM})$ corresponding to the AuNP 15 sample which corresponds to only 1.33 ppm in volume. This decrease of viscosity observed for our INFs is not the usual finding because a majority of INFs present a higher viscosity than the base fluid, but the NP concentration is much higher than ours and the NPs are usually insulators. In recent reviews about the rheology of INFs, it has been published that in some cases, for conducting metal NPs, viscosity decreases. ${ }^{20,21,40,41}$ Direct comparison of our data is not possible because there is no data published about INFs formed by an IL and AuNPs.

The data obtained allow us to establish a direct connection between the diameter of the AuNPs and the viscosity decrease; qualitatively, we observe in Figure 6 that smaller AuNPs show a higher viscosity decrease, but it seems that this effect is not directly related to the external gold surface. Hence, it is much higher for AuNP60 than for AuNP80, their external surfaces being very similar. Finally, note that AuNProd presents a percentage of viscosity reduction between those of AuNP60 and AuNP80, while it presents a AuNP external surface very close to that of AuNP15 (see Table 1). Thus, the rod shape is less effective in increasing the fluidity of the reference sample than the spherical ones. Regarding the temperature behavior, it can be observed in Figure 6 that the viscosity decreases with temperature increases probably due to the fact that thermal agitation weakens the pseudolattice structure of pure ILs. ${ }^{42}$ Note that a protic IL as PAN presents a strong ionic wellordered nanostructure, not broken by the tiny ethanol content of the samples. ${ }^{43}$

3.4. Ionic Conductivity. Temperature-dependence data of ionic conductivity for the reference sample and the different INFs studied are included in Table S1 in the Supporting Information section and plotted in Figure 7. Ionic conductivity

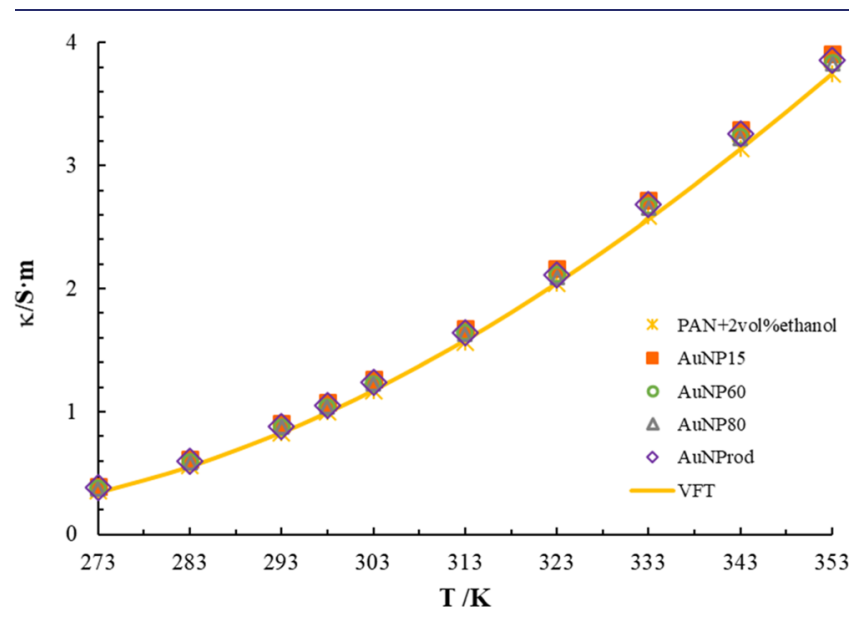

Figure 7. Measured ionic conductivity at different temperatures of reference sample, PAN +2 vol \% ethanol (stars), and different INFs based on PAN-dispersed AuNPs: AuNP15 (square), AuNP60 (circle), AuNP80 (triangle), and AuNProd (rhombus). The line is the best fit of the VFT equation to the reference sample data.

values increase markedly with temperature following a VFT equation for the five samples measured, as was expected. ${ }^{44,45}$ The VFT equation for electrical conductivities is similar to that given in eq 1

$$
\kappa=\kappa_{\infty} \exp \left\{\frac{-B_{\kappa}}{T-T_{\kappa}}\right\}
$$

where again $\kappa_{\infty}, B_{\kappa}$, and $T_{\kappa}$ are fitting parameters. The first one represents the limiting value of $\mathrm{k}$ at infinite temperature, and the second one is related to the activation energy for ion charge transport.

The VFT equation was best-fit to all samples, but we only plot in Figure 7 the one corresponding to the reference one. 
We observe that, in general, the addition of AuNPs to the reference fluid slightly improves its ionic conductivity. To compare the results obtained in the different INFs, we must again normalize the measured values for each sample to a common concentration. As for viscosity, we have chosen that of the AuNP15 sample $(0.13 \mathrm{mM})$. To do that, we simply assume that the deviations of measured $\kappa$ from the reference sample value are proportional to the AuNPs concentration in the range studied. ${ }^{44,45}$ In Figure 8 , we plot the percentage of

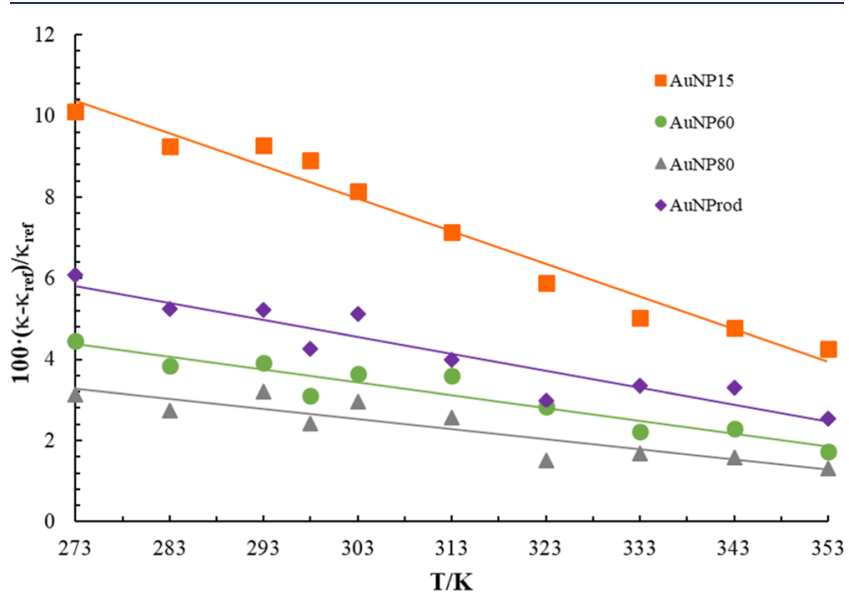

Figure 8. Percentage deviation of ionic conductivity normalized to the same gold concentration of $0.13 \times 10^{-3} \mathrm{~m}$ : AuNP15 (square), AuNP60 (circle), AuNP80 (triangle), and AuNProd (rhombus). The lines are the best fit of the data to a straight line.

ionic conductivity increase for the four different INFs at the common concentration. As also happens for viscosity, the maximum increase is for AuNP15 and the minimum one for AuNP80 (but this last is similar to the other two INFs). With respect to the temperature behavior, the effect of AuNP addition decreases with temperature for all samples but is more evident for that with the highest deviation, like it happens for viscosity, as shown in Figure 6. Hence, while at $273 \mathrm{~K}$, differences among the different samples areare up to $10 \%$, at $353 \mathrm{~K}$, they are about $5 \%$. This fact can be easily explained due to the weakening of the ionic pseudolattice due to an increase in thermal agitation; so, the perturbation produced by the AuNPs is less important. It seems again that the external gold area can explain this result. If AuNPs are solvated by cations, the ionic pseudo-lattice that presents all $\mathrm{ILs}^{46}$ is destroyed in the vicinity of the AuNPs. This fact allows the anions to move faster in this region, thus increasing the net ionic conductivity. In the case of the AuNProd sample, it presents a lower ionic conductivity increase than could be expected from its external surface. To understand this fact, we suppose that the rod shape does not have a privileged orientation with the electrical field, and so it does not favor the ionic mobility along the long axis.

Unfortunately, it is not possible to develop a quantitative model of the effects observed with our present data due to three facts: first, expanded uncertainty of the data presented in Figure 8; second, PAN is a high hygroscopic compound, ${ }^{44}$ therefore the presence of different tiny quantities of water in the different samples would alter the measured values, and third, the calculation of the gold exposed surface is approximate due to the different AuNP sizes in each INF, as observed in the histograms shown in Figure 1. From a qualitative point of view, our results show that even tiny quantities of AuNPs will increase the ionic conductivity up to $10 \%$, that the effect is more pronounced for the smallest of the spherical AuNPs, and that a rod shape is less efficient than a spherical one. This increase of the electrical conductivity with the addition of NPs has been reported before. The effect is more pronounced for metal conducting NPs, but it has been also observed in insulating ones. ${ }^{45}$ Also, it has been reported that the increase of electrical conductivity decreases as the NP concentration increases for non-conducting ones. ${ }^{46}$ The increase in electrical conductivity was found to be mainly determined by three causes: surface conductance of nanoparticles, electrical double layer development, and liquid polarity. ${ }^{45}$ As before, direct comparison with previous data is not possible because there is not any publication with the same INF published earlier, and again, the nature of NPs and base fluid is crucial for the electrical conductivity behavior.

With respect to the temperature behavior, the effect of AuNP addition decreases with temperature for all samples but is more evident for that with the highest $\Delta \kappa$, like it happens for $\Delta \eta$ shown in Figure 6 . Thus, while at $273 \mathrm{~K}$, differences among the different samples are up to $10 \%$, at $353 \mathrm{~K}$, they are about $5 \%$. This fact can be easily explained due to the weakening of the ionic pseudolattice due to an increase in thermal agitation, so the perturbation produced by the AuNPs is less important.

3.5. Walden's Rule. The three magnitudes measured here: density, viscosity, and ionic conductivity $(\rho, \eta$, and $\kappa$, respectively) can be related among them for each sample using Walden's rule, ${ }^{47-49}$ which relates viscosity with molar conductivity, $\Lambda=\kappa / C$ (where $C$ is the ionic concentration of the sample, i.e., $C=\rho / \mathrm{M}$, where $\mathrm{M}$ is the molar mass of PAN) through the following equation

$$
\Lambda \eta=K^{\prime}
$$

where $K^{\prime}$ is a constant called the Walden product. ILs do not follow that simple expression probably due to the fact that the activation energies for conductivity and viscosity are different mainly because of the strong coordination among the ions in ILs and to their great size difference. ${ }^{36}$ Thus, in these systems, we replace eq 3 by the so-called fractional Walden's rule, ${ }^{48,50}$ which reads

$$
\Lambda \eta^{\gamma}=K
$$

This equation takes into account that Arrhenius activation energy for ionic conductivity is lower than that of viscosity. The plot of $\log \Lambda\left(\mathrm{S} \mathrm{cm}^{2} \mathrm{~mol}^{-1}\right)$ versus $\log \eta^{-1}\left(\right.$ Poise $\left.^{-1}\right)$ is known as Walden's plot. This plot provides information about the tendency of ILs to form neutral ion pairs; in other words, the number of mobile charge carriers in an IL. The slope of the Walden plot, $\gamma$, is related to the ratio of activation energies of conductivity and viscosity. Walden's plot takes as a reference the ideal line obtained from a dilute $0.01 \mathrm{M}$ aqueous $\mathrm{KCl}$ solution, where $\log \Lambda\left(\mathrm{S} \mathrm{cm} \mathrm{mol}^{-1}\right)$ is equal to $\log \eta^{-1}$ $\left(\right.$ Poise $\left.^{-1}\right)$ for all ranges of values. In this ideal solution, the ions are fully dissociated and both anion and cation have equal mobility. The deviation of data obtained for any electrolyte from the reference line of $\mathrm{KCl}$ is an indicator of its "degree of ionicity". ${ }^{48,50}$ Thus, in Figure 9, we show the Walden's plot for the four INFs studied and the reference sample, with the corresponding linear fit. As can be observed, the five samples present a linear tendency, which indicates that eq 4 is valid also for these samples. They also present a similar $\gamma$ slope, from 0.90 for AuNP60 to 0.87 for the reference sample, similar to that presented by other ILs, ${ }^{44,46}$ while the $\mathrm{K}$ value ranges from 


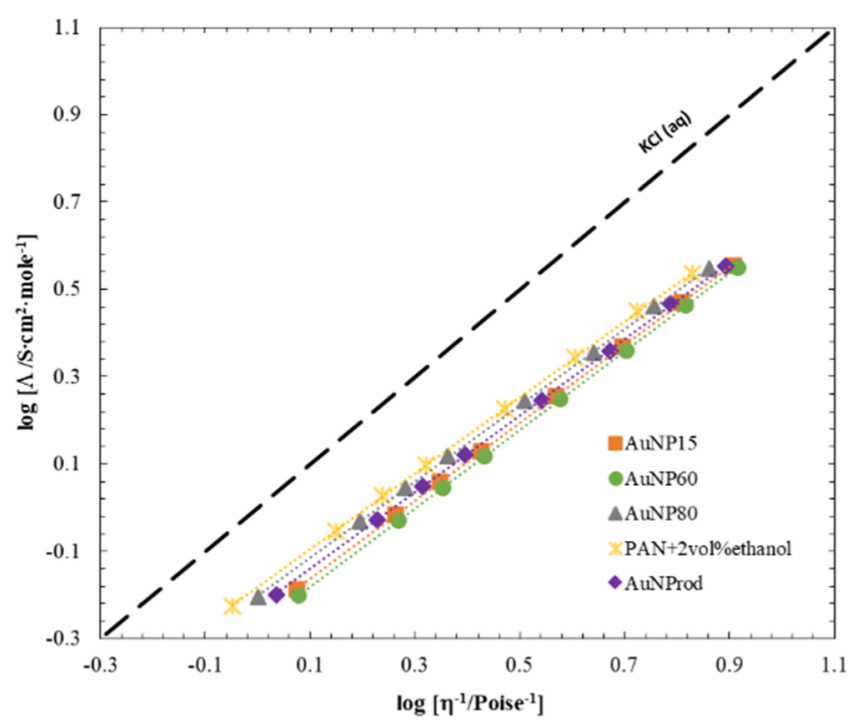

Figure 9. Walden plot for different INFs studied: reference sample (stars), AuNP15 (square), AuNP60 (circle), AuNP80 (triangle), and AuNProd (rhombus).

0.66 to 0.56 , being higher for the samples with smaller $\gamma$ value. The addition of AuNPs to the reference sample produces a decrease in the degree of ionicity indicating that the number of free ions decreases. This is compatible with the solvation of AuNP external surface by the IL cations, which get trapped in the gold surface and so decrease the ionicity. This effect of cations solving the AuNPs has been detected for ILs, ${ }^{34}$ and it has been used to synthesize AuNPs. ${ }^{35}$

From another point of view, the effect of adding AuNPs to the IL is similar to adding a solvent to it, that is, both break the pseudolattice of the IL, allowing more mobility of anions and so increasing fluidity and electrical charge transport. A more quantitative analysis is difficult as explained above, and our next aim is to measure the same sample with different AuNPs content.

\section{CONCLUSIONS}

We have measured the variation of three different physical properties of four INFs composed of the IL PAN plus 2 vol \% ethanol (equivalent to $x_{\mathrm{et}}=0.0365$ ) which contains AuNPs (AuNPs) of three different sizes and of two morphologies in the final concentrations of about $0.15 \mathrm{mM}$ of gold. INFs increase density and ionic conductivity and decrease viscosity with respect to the reference sample or base fluid. This effect is higher for AuNP15. These facts could be explained assuming that propyl ammonium cations solvate the AuNPs' external surface as if they were surfactants; so, density increases due to the packing. Also, the ionic layers covering the AuNPs break the IL network in their vicinity; so, more nitrate anions will be free to move, increasing fluidity and ionic conductivity. From the comparison among the different sizes and shapes studied, we have observed that for smaller AuNPs, the effects are more important (for a fixed gold concentration) and also that the rod shape is less effective than the spherical one to improve conductivity and fluidity in terms of overall $\mathrm{Au}$ external surface in the different INFs studied. Comparison of the results obtained here and those published in the literature for other INFs is impossible because the nature of the NPs and of the base fluid are crucial in the behavior of the studied magnitudes but also, NP size and shape, INF concentration, temperature, and so forth affect the transport properties. In addition, there is not, to our knowledge, any paper devoted to the same (or even similar) INF to those studied here. In any case, more research is needed to fully understand the AuNP-addition effect at a nanoscale and to control quantitatively the effect on density, viscosity, and ionic conductivity of AuNP doping of ILs.

\section{ASSOCIATED CONTENT}

\section{Supporting Information}

The Supporting Information is available free of charge at https://pubs.acs.org/doi/10.1021/acs.jced.1c00135.

Extinction spectra of $60 \mathrm{~nm}$ AuNPs in ethanol and in PAN and densities, viscosities, and electrical conductivities of the reference sample (PAN +2 vol \% ethanol) and those of the four AuNP-added mixtures at atmospheric pressure $(P=101.3 \mathrm{kPa})(\mathrm{PDF})$

\section{AUTHOR INFORMATION}

\section{Corresponding Author}

Oscar Cabeza - Grupo Mesturas, Universidade da Coruña, 15071 A Coruña, Spain; 이이.org/0000-0002-6247-

1161; Phone: +34 981 167000; Email: oscabe@udc.es;

Fax: +34 981167065

\section{Authors}

Sandra García-Garabal - Grupo Mesturas, Universidade da Coruña, 15071 A Coruña, Spain

Montserrat Domínguez-Pérez - Grupo Mesturas, Universidade da Coruña, 15071 A Coruña, Spain

Yago Arosa - NAFOMAT, Universidad de Santiago de Compostela, 15782 Santiago de Compostela, Spain

Luis Miguel Varela - NAFOMAT, Universidad de Santiago de Compostela, 15782 Santiago de Compostela, Spain; (1) orcid.org/0000-0002-0569-0042

Cristina Fernández-López - CINBIO, Departamento de Química Física, Universidade de Vigo, 36310 Vigo, Spain

Jorge Pérez-Juste - CINBIO, Departamento de Química Física, Universidade de Vigo, 36310 Vigo, Spain; () orcid.org/0000-0002-4614-1699

Isabel Pastoriza-Santos - CINBIO, Departamento de Química Física, Universidade de Vigo, 36310 Vigo, Spain; ○ orcid.org/0000-0002-1091-1364

Complete contact information is available at: https://pubs.acs.org/10.1021/acs.jced.1c00135

\section{Author Contributions}

This manuscript was written through the contributions of all authors. All authors have given approval to the final version of the manuscript. Researchers from CINBIO performed the AuNP synthesis and preparation, while experimental measurements and data analysis were done by Mesturas and NAFOMAT teams.

\section{Funding}

Spanish "Ministerio de Economía y Competitividad (MINECO)” (MAT2016-77809-R, MAT2017-89239-C2-2-P, and MAT2017-89239-C2-1-P) and "Xunta de Galicia" (GRC ED431C 2016/001 and ED431D 2017/06) are acknowledged.

\section{Notes}

The authors declare no competing financial interest. 


\section{ACKNOWLEDGMENTS}

We acknowledge UDC technician D. Manuel Cabanas for some of the measurements presented here. The financial support of Ministerio de Economía y Competitividad (MINECO) (MAT2016-77809-R and MAT2017-89239-C2(1,2)-P); Xunta de Galicia (GRC ED431C 2016/001 and ED431D 2017/06) is also acknowledged. All these research projects were partially supported by FEDER.

\section{REFERENCES}

(1) Das, S. K.; Choi, S. U. S.; Patel, H. E. Heat Transfer in Nanofluids-A Review. Heat Transfer Eng. 2006, 27, 3-19.

(2) Wang, X.-Q.; Mujumdar, A. S. Heat transfer characteristics of nanofluids: a review. Int. J. Therm. Sci. 2007, 46, 1-19.

(3) Mitra, S.; Saha, S. K.; Chakraborty, S.; Das, S. Study on boiling heat transfer of water- $\mathrm{TiO}_{2}$ and water-MWCNT nanofluids based laminar jet impingement on heated steel surface. Appl. Therm. Eng. 2012, 37, 353-359.

(4) Mahian, O.; Kianifar, A.; Kalogirou, S. A.; Pop, I.; Wongwises, S. A review of the applications of nanofluids in solar energy. Int. J. Heat Mass Transfer 2013, 57, 582-594.

(5) Verma, S. K.; Tiwari, A. K. Progress of nanofluid application in solar collectors: A review. Energy Convers. Manage. 2015, 100, 324346.

(6) Paul, T. C.; Morshed, A. K. M. M.; Khan, J. A. Effect of Nanoparticle Dispersion on Thermophysical Properties of Ionic Liquids for its Potential Application in Solar Collector. Procedia Eng. 2014, 90, 643-648.

(7) De, M.; Ghosh, P. S.; Rotello, V. M. Applications of Nanoparticles in Biology. Adv. Mater. 2008, 20, 4225-4241.

(8) Nguyen, K. T.; Zhao, Y. Engineered Hybrid Nanoparticles for On-Demand Diagnostics and Therapeutics. Acc. Chem. Res. 2015, 48, 3016-3025.

(9) Kwak, K.; Kumar, S. S.; Pyo, K.; Lee, D. Ionic Liquid of a Gold Nanocluster: A Versatile Matrix for Electrochemical Biosensors. ACS Nano 2014, 8, 671-679.

(10) Huang, X.; Jain, P. K.; El-Sayed, I. H.; El-Sayed, M. A. Gold nanoparticles: interesting optical properties and recent applications in cancer diagnostics and therapy. Nanomedicine 2007, 2, 681-693.

(11) Santos, C. E. A.; Alencar, M. A. R. C.; Oliveira, L. F.; Scheeren, C. W.; Dupont, J.; Hickmann, J. M. Colloidal metallic nanoparticles in ionic liquids: new systems for nonlinear optical applications. Frontiers in Optics 2009/Laser Science XXV/Fall 2009 OSA Optics \& Photonics Technical Digest, Advances in Optical Materials; San Jose, California: USA, 2009.

(12) Gómez, L. A.; de Araújo, C. B.; Galembeck, A.; Galembeck, A. Solvent effects on the linear and nonlinear optical response of silver nanoparticles. Appl. Phys. B 2008, 92, 61-66.

(13) Choi, S. U. S. Nanofluids From Vision to Reality Through Research. J. Heat Transfer 2009, 131, 033106.

(14) Liu, J.; Wang, F.; Zhang, L.; Fang, X.; Zhang, Z. Thermodynamic properties and thermal stability of ionic liquidbased nanofluids containing graphene as advanced heat transfer fluids for medium-to-high-temperature applications. Renewable Energy 2014, $63,519-523$.

(15) Wang, B.; Wang, X.; Lou, W.; Hao, J. Ionic liquid-based stable nanofluids containing gold nanoparticles. J. Colloid Interface Sci. 2011, $362,5-14$.

(16) Vajjha, R. S.; Das, D. K. A review and analysis on influence of temperature and concentration of nanofluids on thermophysical properties, heat transfer and pumping power. Int. J. Heat Mass Transfer 2012, 55, 4063-4078.

(17) Wang, B.; Wang, X.; Lou, W.; Hao, J. Gold-ionic liquid nanofluids with preferably tribological properties and thermal conductivity. Nanoscale Res. Lett. 2011, 6, 259-269.

(18) Nabati Shoghl, S.; Jamali, J.; Keshavarz Moraveji, M. Electrical conductivity, viscosity, and density of different nanofluids: An experimental study. Exp. Therm. Fluid Sci. 2016, 74, 339-346.
(19) Pastoriza-Gallego, M. J.; Casanova, C.; Legido, J. L.; Piñeiro, M. $\mathrm{M}$. $\mathrm{CuO}$ in water nanofluid: Influence of particle size and polydispersity on volumetric behaviour and viscosity. Fluid Phase Equilib. 2011, 300, 188-196.

(20) Murshed, S. M. S.; Estellé, P. A state of the art review on viscosity of nanofluids. Renew. Sustain. Energy Rev. 2017, 76, 11341152.

(21) Hozwiak, B.; Boncel, S. Rheology of ionanofluids - A review. J. Mol. Liq. 2020, 302, 112568.

(22) Ganguly, S.; Sikdar, S.; Basu, S. Experimental investigation of the effective electrical conductivity of aluminum oxide nanofluids. Powder Technol. 2009, 196, 326-330.

(23) Nieto de Castro, C. A.; Ribeiro, A. P. C.; Vieira, S. I. C.; França, J. M. P.; Lourenço, M. J. V.; Santos, F. V.; Murshed, S. M. S.; Goodrich, P.; Hardacre, C. Ionic Liquids: New Aspects for the Future; Kadokawa, J., Eds.; InTech, 2013; Chapter 7.

(24) Hallinan, D. T.; Balsara, N. P. Polymer electrolytes. Annu. Rev. Mater. Res. 2013, 43, 503-525.

(25) He, Z.; Alexandridis, P. Ionic liquid and nanoparticle hybrid systems: Emerging applications. Adv. Colloid Interface Sci. 2017, 244, 54-70.

(26) Lu, Y.; Korf, K.; Kambe, Y.; Tu, Z.; Archer, L. A. Ionic-liquidnanoparticle hybrid electrolytes: applications in lithium metal batteries. Angew. Chem., Int. Ed. Engl. 2014, 53, 488-492.

(27) Zhao, Y.; Boström, T. Ionic liquid and nanoparticle based magnetic electrolytes: Design, preparation, and electrochemical stability characterization. J. Mol. Liq. 2016, 213, 268-272.

(28) Zhou, R.; Liu, W.; Yao, X.; Leong, Y. W.; Lu, X. Poly(vinylidene fluoride) nanofibrous mats with covalently attached $\mathrm{SiO}_{2}$ nanoparticles as an ionic liquid host: enhanced ion transport for electrochromic devices and lithium-ion batteries. J. Mater. Chem. A 2015, 3, 16040-16049.

(29) He, Z.; Alexandridis, P. Nanoparticles in ionic liquids: Interactions and organization. Phys. Chem. Chem. Phys. 2015, 17, $18238-18261$.

(30) Turkevich, J.; Stevenson, P. C.; Hillier, J. A study of the nucleation and growth processes in the synthesis of colloidal gold. Discuss. Faraday Soc. 1951, 11, 55-75.

(31) Rodríguez-Fernández, J.; Pérez-Juste, J.; García de Abajo, F. J.; Liz-Marzán, L. M. Seeded growth of submicron Au colloids with quadrupole plasmon resonance modes. Langmuir 2006, 22, 70077010.

(32) Nikoobakht, B.; El-Sayed, M. A. Preparation and Growth Mechanism of Gold Nanorods (NRs) Using Seed-Mediated Growth Method. Chem. Mater. 2003, 15, 1957-1962.

(33) Prego, M.; Rilo, E.; Carballo, E.; Franjo, C.; Jiménez, E.; Cabeza, O. Electrical conductivity data of alkanols from 273 to $333 \mathrm{~K}$. J. Mol. Liq. 2003, 102, 83-91.

(34) Dash, P.; Scott, R. W. J. 1-Methylimidazole stabilization of gold nanoparticles in imidazolium ionic liquids. Chem. Commun. 2009, 7, 812-814.

(35) Chen, S.; Liu, Y.; Wu, G. Stabilized and size-tunable gold nanoparticles formed in a quaternary ammonium-based roomtemperature ionic liquid under $\gamma$-irradiation. Nanotechnology 2005, 16, 2360-2364.

(36) García-Garabal, S.; Vila, J.; Rilo, E.; Domínguez-Pérez, M.; Segade, L.; Tojo, E.; Verdía, P.; Varela, L. M.; Cabeza, O. Transport properties for 1-ethyl-3-methylimidazolium n-alkyl sulfates: possible evidence of Grotthuss mechanism. Electrochim. Acta 2017, 231, 94102.

(37) Vogel, H. The law of the relationship between viscosity of liquids and the temperature. Phys. Z. 1921, 19, 645-646.

(38) Fulcher, G. S. Analysis of recent measurements of the viscosity of glasses. J. Am. Ceram. Soc. 1925, 8, 339-355.

(39) Tammam, G.; Hesse, W.; Anorg, Z. Die Abhängigkeit der Viscosität von der Temperatur bie unterkühlten Flüssigkeiten. $Z$. Anorg. Allg. Chem. 1926, 156, 245-257. 
(40) Namburu, P. K.; Kulkarni, D. P.; Misra, D.; Das, D. K. Viscosity of copper oxide nanoparticles dispersed in ethylene glycol and water mixture. Exp. Therm. Fluid Sci. 2007, 32, 397-402.

(41) Yang, J.-C.; Li, F.-C.; Zhou, W.-W.; He, Y.-R.; Jiang, B.-C. Experimental investigation on the thermal conductivity and shear viscosity of viscoelastic-fluid-based nanofluids. Int. J. Heat Mass Transfer 2012, 55, 3160-3166.

(42) Varela, L. M.; Carrete, J.; García, M.; Rodríguez, J. R.; Turmine, M.; Cabeza, O. Ionic Liquids Theory, Properties, New Approaches; Kokorin, A., Ed.; InTech, 2011.

(43) Segade, L.; Cabanas, M.; Domínguez-Pérez, M.; Rilo, E.; García-Garabal, S.; Turmine, M.; Varela, L. M.; Gómez-González, V.; Docampo-Alvarez, B.; Cabeza, O. Surface and bulk characterisation of mixtures containing alkylammonium nitrates and water or ethanol: Experimental and simulated properties at 298.15 K. J. Mol. Liq. 2016, 222, 663-670.

(44) Vila, J.; Ginés, P.; Rilo, E.; Cabeza, O.; Varela, L. M. Great increase of the electrical conductivity of ionic liquids in aqueous solutions. Fluid Phase Equilib. 2006, 247, 32-39.

(45) Minea, A. A. Review on Electrical Conductivity of Nanoparticle-Enhanced Fluids. Nanomaterials 2019, 9, 1592.

(46) Chereches, E. I.; Minea, A. A. Experimental evaluation of electrical conductivity of ionanofluids based on water- $\left[\mathrm{C}_{2} \mathrm{mim}\right]$ $\left[\mathrm{CH}_{3} \mathrm{SO}_{3}\right]$ ionic liquids mixtures and alumina nanoparticles. J. Therm. Anal. Calorim. 2020, DOI: 10.1007/s10973-020-09925-z.

(47) Angell, C. A.; Byrne, N.; Belieres, J.-P. Parallel Developments in Aprotic and Protic Ionic Liquids: Physical Chemistry and Applications. Acc. Chem. Res. 2007, 40, 1228-1236.

(48) Xu, W.; Cooper, E. I.; Angell, C. A. Ionic Liquids: Ion Mobilities, Glass Temperatures, and Fragilities. J. Phys. Chem. B 2003, 107, 6170-6178.

(49) Yoshizawa, M.; Xu, W.; Angell, C. A. Ionic Liquids by Proton Transfer: Vapor Pressure, Conductivity, and the Relevance of $\Delta \mathrm{pK}_{\mathrm{a}}$ from Aqueous Solutions. J. Am. Chem. Soc. 2003, 125, 15411-15419. (50) Austen Angell, C.; Ansari, Y.; Zhao, Z. Ionic Liquids: Past, present and future. Faraday Discuss. 2012, 154, 9-27. 